\title{
Adaptive Antenna Assisted Network Performance of FDD-mode UMTS
}

\author{
J.S. Blogh, L. Hanzo \\ Dept. of Electronics and Computer Science, Univ. of Southampton, SO17 1BJ, UK. \\ Tel: +44-2380-593 125, Fax: +44-2380-593 045 \\ Email: Ih@ecs.soton.ac.uk; http://www-mobile.ecs.soton.ac.uk
}

\begin{abstract}
The performance of the Frequency Division Duplex (FDD) mode of the Code Division Multiple Access (CDMA) based Universal Mobile Telecommunication System (UMTS) is investigated. The new call blocking and call dropping probabilities, the probability of low-quality access as well as the required average transmit power are quantified both with and without the assistance of adaptive antenna arrays as well as with and without encountering shadow fading. In some of the scenarios investigated the system's user capacity is doubled with the advent of adaptive antennas.
\end{abstract}

\section{INTRODUCTION AND SOFT HANDOVERS}

With the imminent roll-out of the third-generation (3G) IMT2000 system's UMTS Terrestrial Radio Access (UTRA) mode [1-3] the achievable network performance has to be quantified. In our previous investigations we endeavoured to identify the soft handover algorithm, which supports the highest number of users, at the best call quality, regardless of the propagation conditions [4].

Employing relative received pilot power based handover thresholds is important in realistic propagation environments exposed to shadow fading. More explicitly, in contrast to using absolute handover thresholds, which were expressed in terms of $\mathrm{dBm}$, i.e. with respect to $1 \mathrm{~mW}$ in [4], we also investigated the employment of a pair of relative handover thresholds. Accordingly, both the call acceptance threshold $T_{a c c}$ and the call dropping threshold $T_{d r o p}$ were expressed in terms of $\mathrm{dB}$ relative to the received pilot strength of the base stations in the Active Basestation Set (ABS). The employment of these relative thresholds also caters for situations, where the absolute pilot power may be too low for use in conjunction with fixed thresholds, but nonetheless sufficiently high for reliable communications.

Another soft handover activation metric that we used in [4] for determining "cell ownership" was the pilot to downlink interference power ratio of a cell, which we denoted by $E_{c} / I_{o}$. This handover metric was proposed for employment in the 3rd generation systems [9]. The pilot to downlink interference ratio, or $E_{c} / I_{o}$, may be calculated thus as [10]:

$$
\frac{E_{c}}{I_{o}}=\frac{P_{\text {pilot }}}{P_{\text {pilot }}+N_{0}+\sum_{k=1}^{N_{\text {cells }}} P_{k} T_{k}},
$$

where $P_{k}$ is the total transmit power of cell $k, T_{k}$ is the transmission gain which includes antenna gain and pathloss as well as shadowing, $N_{0}$ is the thermal noise and $N_{\text {cells }}$ is the number of cells in the network. The advantage of using such a scheme

The financial support of the following organisations is gratefully acknowledged: Engineering and Physical Sciences Research Council, Swindon, UK. is that it is not an absolute measurement that is used, but the ratio of the pilot power to the interference power. Thus, if fixed thresholds were used, a form of admission control may be employed for new calls if the interference level became excessive. A further advantage of this technique is that it takes into account the time-varying nature of the interference level in a shadowed environment.

In [4] we concluded that it was beneficial to combine the employment of the received $E_{c} / I_{o}$ ratio and the relative soft handover thresholds, thus ensuring that variations in both the received pilot signal strength and in the interference levels were monitored during the soft handover process.

Since in [4] we identified an attractive handover algorithm, in this contribution we focus our attention on the impact of adaptive antenna arrays on the UTRA network's performance in a pedestrian scenario. Specifically, our investigations were conducted using the relative $E_{c} / I_{o}$ based soft handover algorithm in conjunction with $T_{a c c}=-10 \mathrm{~dB}$ and $T_{d r o p}=-18 \mathrm{~dB}$, using a CDMA spreading factor of 16 . Given that the chip rate of UTRA is $3.84 \mathrm{Mchips} / \mathrm{sec}$, this spreading factor corresponds to a channel data rate of $3.84 \times 10^{6} / 16=240 \mathrm{kbps}$. Applying $1 / 2$ rate error correction coding would result in an effective data throughput of $120 \mathrm{kbps}$, whereas utilising a $2 / 3$ rate error correction code would provide a useful throughput of $160 \mathrm{kps}$. A cell radius of $150 \mathrm{~m}$ was assumed and a pedestrian walking velocity of $3 \mathrm{mph}$ was used. The remaining system characteristics - including the power control scheme, the Orthogonal Variable Spreading Factor (OVSF) code allocation algorithm [2] and the multi-user detector [5] - were identical to those used in [4], which are also summarised in Table I.

\section{Performance Metrics}

The following network performance metrics have been employed in our investigations [6]:

- New call blocking probability, $P_{B}$.

- Call dropping or forced termination probability, $P_{F T}$. A call is dropped when the lower of the uplink and downlink SINRs dips consecutively below the outage SINR (1\% BER) a given number of times.

- Probability of a low quality access, $P_{l o w}$, quantifies the chances of either the uplink or downlink signal quality being sufficiently poor, resulting in a low quality access (0.5\% BER).

- Probability of outage, $P_{\text {out }}$, is defined as the probability that the SINR is below the value at which the call is deemed to be in outage.

- Grade-Of-Service (GOS) was defined by Cheng and 


\begin{tabular}{|l|r||l|r|} 
rarameter & value & rarameter & value \\
\hline \hline Noisefloor & $-100 \mathrm{dBm}$ & Pilot power & $-5 \mathrm{dBm}$ \\
Frame length & $10 \mathrm{~ms}$ & Cell radius & $150 \mathrm{~m}$ \\
Multiple access & FDD/CDMA & Number of basestations & 49 \\
Modulation scheme & 4QAM/QPSK & Spreading factor & 16 \\
Minimum BS transmit power & $-44 \mathrm{dBm}$ & Minimum MS transmit power & $-44 \mathrm{dBm}$ \\
Maximum BS transmit power & $21 \mathrm{dBm}$ & Maximum MS transmit power & $21 \mathrm{dBm}$ \\
Power control stepsize & $1 \mathrm{~dB}$ & Power control hysteresis & $1 \mathrm{~dB}$ \\
Low quality access (0.5\% BER) SINR & $7.0 \mathrm{~dB}$ & Outage (1\% BER) SINR & $6.6 \mathrm{~dB}$ \\
Pathloss exponent & -3.5 & Size of Active Basestation Set (ABS) & 2 \\
Average inter-call-time & $300 \mathrm{~s}$ & Max. new-call queue-time & $5 \mathrm{~s}$ \\
Average call length & $60 \mathrm{~s}$ & Pedestrian speed & $3 \mathrm{mph}$ \\
Maximum consecutive outages & 5 & Signal bandwidth & $5 \mathrm{MHz}$ \\
Target SINR (at BER=0.1\%) & $8.0 \mathrm{~dB}$ & & \\
\hline
\end{tabular}

TABLE I

Simulation parameters.

Chuang [6] as :

$$
\begin{aligned}
G O S= & P\{\text { unsuccessful or low-quality call accesses }\} \\
= & P\{\text { call is blocked }\}+P\{\text { call is admitted }\} \times \\
& P\{\text { low signal quality and call is admitted }\} \\
= & P_{B}+\left(1-P_{B}\right) P_{\text {low }} .
\end{aligned}
$$

In order to determine the number of users that may be supported with adequate call quality by the network, we have defined a conservative and a lenient scenario, which are created from a combination of the performance metrics, as follows [7,8]:

- Conservative scenario :

$P_{B} \leq 3 \%, P_{F T} \leq 1 \%, P_{\text {low }} \leq 1 \%$ and $G O S \leq 4 \%$.

- Lenient scenario :

$P_{B} \leq 5 \%, P_{F T} \leq 1 \%, P_{\text {low }} \leq 2 \%$ and $G O S \leq 6 \%$.

\section{Network Performance}

In our previous investigations employing adaptive antenna arrays at the base station [7] we observed quite significant performance gains as a direct result of the interference rejection capabilities of the adaptive antenna arrays invoked. Since the CDMA based network considered here has a frequency reuse of 1 , the levels of co-channel interference are significantly higher than in [7], and hence the adaptive antennas may be able to null the interference more effectively. However, the high number of interference sources may limit the interference rejection achievable with the aid of a limited number of array elements.

Network performance results were obtained using two and four element adaptive antenna arrays, both in the absence of shadow fading, and in the presence of $0.5 \mathrm{~Hz}$ and $1.0 \mathrm{~Hz}$ frequency shadow fading exhibiting a standard deviation of $3 \mathrm{~dB}$. The adaptive beamforming algorithm used was the Sample Matrix Inversion (SMI) algorithm. The specific adaptive beamforming implementation used in the CDMA based network was identical to that used in the FDMA/TDMA network simulations of [7].
Briefly, one of the eight possible 8-bit BPSK reference signals was used for identifying the desired user, while the remaining interfering users were assigned the other seven 8-bit reference signals. The received signal's autocorrelation matrix was then calculated, and from the knowledge of the desired user's reference signal the receiver's optimal antenna array weights were determined with the aid of the SMI algorithm. Since this implementation of the algorithm only calculated the receiver's antenna array weights, i.e. the antenna array weights used by the base station for receiving the mobiles' uplink transmissions, these weights may not be suitable for use in the downlink, when independent up/downlink shadow fading is experienced. Hence, further investigations were conducted, where the uplink and downlink channels were identical, in order to determine the potential performance gain that may be achieved by separately calculating the antenna array weights to be used in the downlink. The antenna array weights were re-calculated for every power control step, i.e. 15 times per UTRA data frame, due to the potential significant changes in terms of the desired signal and interference powers that may occur during one UTRA frame as a result of the maximum possible $15 \mathrm{~dB}$ change in the power transmitted by each user.

Figure 1 shows the significant reduction in the probability of a dropped call achieved by employing adaptive antenna arrays in a non-shadowed propagation environment. The figure has demonstrated that even with only two antenna elements, the adaptive antenna arrays have considerably reduced the levels of co-channel interference, leading to a reduced call dropping probability. This has been achieved in spite of the numerous sources of co-channel interference resulting from the frequency reuse factor of one, which was remarkable in the light of the limited number of degrees of freedom of the two element array. Without employing antenna arrays at the base stations the network capacity was limited to 256 users, or to a teletraffic load of approximately 1.4 Erlangs $/ \mathrm{km}^{2} / \mathrm{MHz}$. However, with the advent of two element adaptive antenna arrays at the base stations the number of users supported by the network rose by $27 \%$ to 325 users, or almost 1.9 Erlangs $/ \mathrm{km}^{2} / \mathrm{MHz}$. Replacing the two 


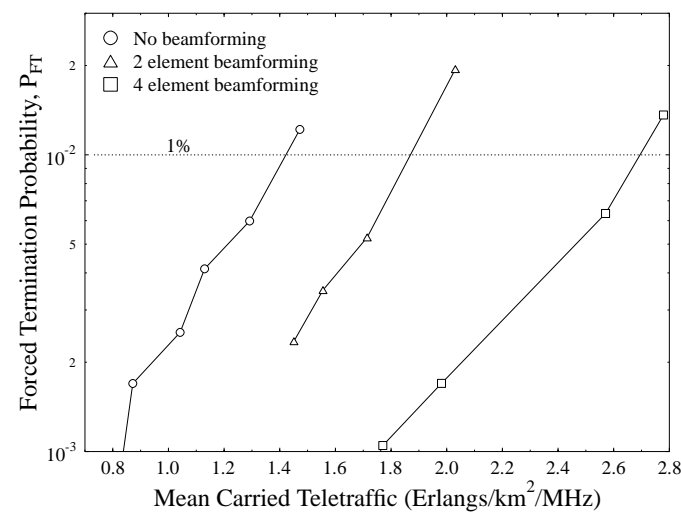

Fig. 1. Call dropping probability versus mean carried traffic of a CDMA based cellular network using relative received $E_{c} / I_{o}$ based soft handover thresholds with and without beamforming and without shadowing for $\mathrm{SF}=16$.

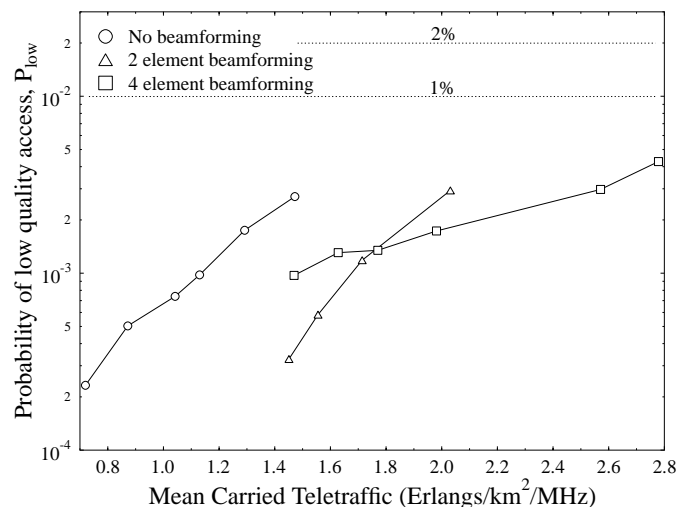

Fig. 2. Probability of low quality access versus mean carried traffic of a CDMA based cellular network using relative received $E_{c} / I_{o}$ based soft handover thresholds with and without beamforming and without shadowing for $\mathrm{SF}=16$.

element adaptive antenna arrays with four element arrays led to a further rise of $48 \%$, or $88 \%$ with respect to the capacity of the network using no antenna arrays. This is associated with a network capacity of 480 users, or 2.75 Erlangs $/ \mathrm{km}^{2} / \mathrm{MHz}$. A summary of the network capacities achieved under different conditions is given in Table II.

The probability of low quality outage, presented in Figure 2 also exhibited a substantial improvement with the advent of two element adaptive antenna arrays. However, the performance trends associated with invoking four element adaptive antenna arrays required further interpretations. Specifically, it can be seen from the figure that higher traffic loads were carried with the aid of the four-element array a sufficiently low probability of a low quality occurring. By contrast, at higher traffic loads the probability of a low quality access was lower than that achieved using a two element array. However, again, at lower traffic loads the performance was worse than that obtained when using two element arrays, and the gradient of the performance curve was significantly lower. Further in-depth analysis of the results not included here due to lack of space suggested that the vast majority of the low quality outages were occurring when new calls started. When a user decided to commence communications with the base station, the current interference level was measured, and the target transmission power was determined in order to reach the target SINR necessary for reliable communications. However, in order to avoid disrupting existing calls the transmission power was ramped up slowly, until the target SINR was reached. A network using no adaptive antenna arrays, i.e. employing omnidirectional antennas, can be viewed as offering equal gain to all users of the network, which we assumed to be 1.0 , or $0 \mathrm{~dB}$. Thus, when a new call is initiated, the level of interference rises gradually and the power control algorithm ensures that the existing users compensate for the increased level of cochannel interference by increasing their transmission power. In a network using adaptive antenna arrays, the adaptive antenna arrays are used for nulling the sources of interference and in doing so the array may reduce the antenna gain in the direction of the desired user, in order to maximise the SINR. Hence a user initiating a new call, even if it has a low transmission power, can alter the antenna array's response, thus altering the antenna gain experienced by the existing users. This phenomenon is more marked, when using four element arrays, since their directivity, and thus sensitivity to interfering signals, is higher.

Even though the employment of adaptive antenna arrays may result in the attenuation of the desired signal, this is performed in order to maximise the received SINR, and thus the levels of interference are attenuated more strongly, ultimately leading to the reduction of the mean transmission power, as emphasised by Figure 3. This figure clearly shows the lower levels of transmission power, required in order to maintain an acceptable performance, whilst using adaptive antenna arrays at the base stations. A reduction of $3 \mathrm{~dB}$ in the mean mobile transmission power was achieved by invoking two element antenna arrays, and a further reduction of $1.5 \mathrm{~dB}$ resulted from using four element arrays. These power budget savings were obtained in conjunction with reduced levels of co-channel interference, leading to superior call quality, as illustrated in Figures 1 and 2. A higher performance advantage was evident in the uplink scenario, suggesting that the selective base station diversity techniques employed in the uplink are amenable to amalgamation with adaptive antenna arrays. By contrast, the maximum ratio combining performed at the mobile inherently reduces the impact of co-channel interference, and hence benefits to a lesser extent from the employment of adaptive antenna arrays.

The impact of adaptive antenna arrays in a propagation environment subjected to shadow fading was then investigated. The associated call dropping performance is shown in Figure 4. This figure illustrates the substantial network capacity gains achieved with the aid of both two and four element adaptive antenna arrays under shadow fading propagation conditions. Simulations were conducted in conjunction with log-normal shadow fading having a standard deviation of $3 \mathrm{~dB}$, experiencing maximum shadowing frequencies of both $0.5 \mathrm{~Hz}$ and $1.0 \mathrm{~Hz}$. As expected, the network capacity was reduced at the higher shadow fading frequency. The effect of performing independent up- and down-link beamforming, as opposed to using the base station's receive antenna array weights in the downlink was also studied, and a small, but not insignificant call dropping probability reduction can be seen in the Figure 4. The network supported 


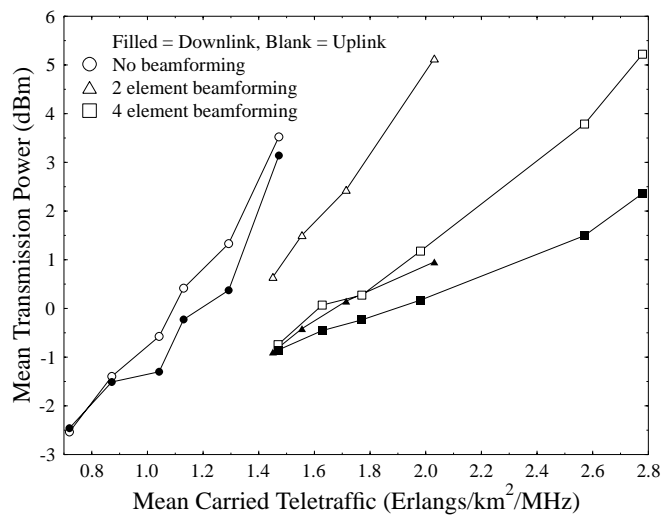

Fig. 3. Mean transmission power versus mean carried traffic of a CDMA based cellular network using relative received $E_{c} / I_{o}$ based soft handover thresholds with and without beamforming and without shadowing for $\mathrm{SF}=16$.

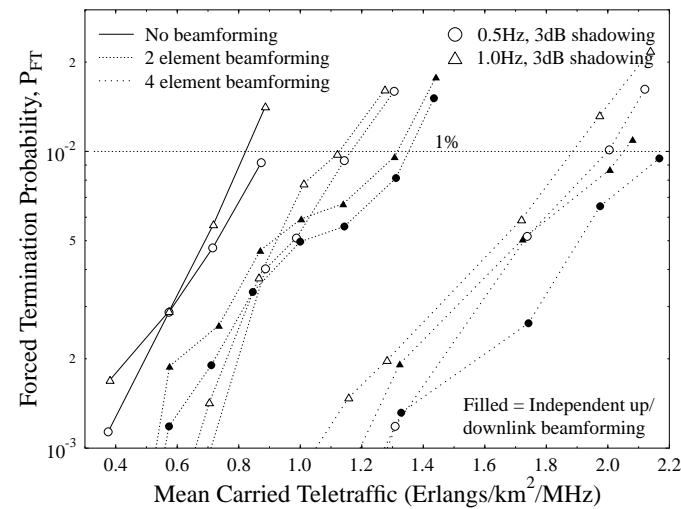

Fig. 4. Call dropping probability versus mean carried traffic of a CDMA based cellular network using relative received $E_{c} / I_{o}$ based soft handover thresholds with and without beamforming and with shadowing having a standard deviation of $3 \mathrm{~dB}$ for $\mathrm{SF}=16$.

just over 150 users and 144 users, when subjected to $0.5 \mathrm{~Hz}$ and $1.0 \mathrm{~Hz}$ frequency shadow fading, respectively. With the application of two element adaptive antenna arrays, re-using the base station's uplink receiver weights on the downlink, these capacities increased by $35 \%$ and $40 \%$, to 203 users and 201 users. Performing independent up- and down-link beamforming resulted in a mean further increase of $13 \%$ in the network capacity. The implementation of four element adaptive antenna arrays led to a network capacity of 349 users at a $0.5 \mathrm{~Hz}$ shadowing frequency, and 333 users at a $1.0 \mathrm{~Hz}$ shadowing frequency. This corresponded to relative gains of $133 \%$ and $131 \%$ over the capacity provided without beamforming. Invoking independent up- and down-link beamforming produced another network capacity enhancement of $7 \%$ and $10 \%$ for the $0.5 \mathrm{~Hz}$ and $1.0 \mathrm{~Hz}$ frequency shadowing environments, respectively, giving final network capacities of just over 375 users and 365 users.

Similar trends were observed regarding the probability of low quality outage to those found in the non-shadowing scenarios. However, the trend was significantly more prevalent under shadowing, due to the higher variation of the received signal strengths, as a result of the shadow fading, as shown in Fig-

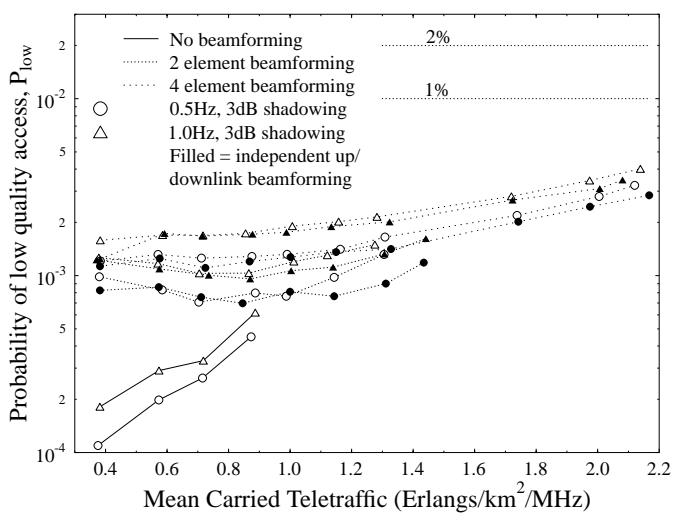

Fig. 5. Probability of low quality access versus mean carried traffic of a CDMA based cellular network using relative received $E_{c} / I_{o}$ based soft handover thresholds with and without beamforming and with shadowing having a standard deviation of $3 \mathbf{~ d B}$ for $\mathrm{SF}=16$.

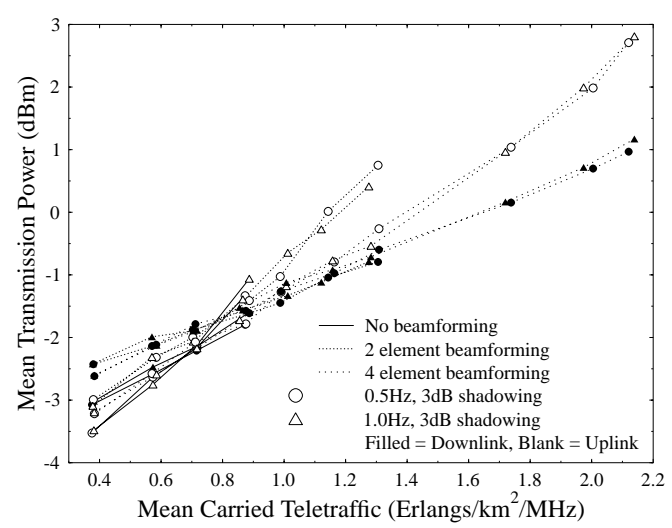

Fig. 6. Mean transmission power versus mean carried traffic of a CDMA based cellular network using relative received $E_{c} / I_{o}$ based soft handover thresholds with and without beamforming and shadowing having a standard deviation of $3 \mathbf{~ d B}$ for $\mathrm{SF}=16$.

ure 5. The figure indicates that the trend is also evident, when using two element adaptive antenna arrays in conjunction with shadow fading. As expected, the performance deteriorated as the number of antenna elements increased, and when the maximum shadow fading frequency was increased from $0.5 \mathrm{~Hz}$ to $1.0 \mathrm{~Hz}$. It should be noted, however that the probability of low quality access always remained below the $1 \%$ constraint of the conservative scenario, and the call dropping probability was considerably reduced by the adaptive antenna arrays.

The mean transmission power performance is depicted in Figure 6 , suggesting that similarly to the non-shadowing scenario of Figure 3, the number of antenna elements had only a limited impact on the base stations' transmission power, although there was some reduction in the mobile stations' mean transmission power. The mean transmission powers required when using independent up- and down-link beamforming are not explicitly shown, but these were slightly less than those presented here, with a mean reduction of about $0.4 \mathrm{~dB}$.

A summary of the maximum user capacities of the networks considered in this section both with and without shadowing, em- 


\begin{tabular}{|c|c|c|c|c|c|c|}
\hline \multirow{3}{*}{ Shadowing } & \multirow{3}{*}{ Beamforming: } & \multirow{3}{*}{$\begin{array}{l}\text { independent } \\
\text { up/down-link }\end{array}$} & \multicolumn{4}{|c|}{ Conservative scenario, $r_{F T}=1 \%, r_{\text {low }}=1 \%$} \\
\hline & & & Users & Traffic (Erlangs & Pow & dBm) \\
\hline & & & & $\left./ \mathrm{km}^{2} / \mathrm{MHz}\right)$ & MS & BS \\
\hline No & No & - & 256 & 1.42 & 3.1 & 2.7 \\
\hline No & 2 elements & - & 325 & 1.87 & 3.75 & 0.55 \\
\hline No & 4 elements & - & 480 & 2.75 & 4.55 & 1.85 \\
\hline$\overline{0.5 \mathrm{~Hz}, 3 \mathrm{~dB}}$ & No & - & $\approx 150$ & 0.87 & 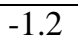 & -1.7 \\
\hline $0.5 \mathrm{~Hz}, 3 \mathrm{~dB}$ & 2 elements & No & 203 & 1.16 & 0.1 & -1.1 \\
\hline $0.5 \mathrm{~Hz}, 3 \mathrm{~dB}$ & 4 elements & No & 349 & 2.0 & 2.0 & 0.65 \\
\hline $0.5 \mathrm{~Hz}, 3 \mathrm{~dB}$ & 2 elements & Yes & 233 & 1.35 & 0.2 & -0.8 \\
\hline $0.5 \mathrm{~Hz}, 3 \mathrm{~dB}$ & 4 elements & Yes & $\approx 375$ & 2.2 & 2.15 & 0.85 \\
\hline $1.0 \mathrm{~Hz}, 3 \mathrm{~dB}$ & No & - & 144 & 0.82 & -1.1 & -1.6 \\
\hline $1.0 \mathrm{~Hz}, 3 \mathrm{~dB}$ & 2 elements & No & 201 & 1.12 & -0.3 & -1.1 \\
\hline $1.0 \mathrm{~Hz}, 3 \mathrm{~dB}$ & 4 elements & No & 333 & 1.88 & 1.6 & 0.5 \\
\hline $1.0 \mathrm{~Hz}, 3 \mathrm{~dB}$ & 2 elements & Yes & 225 & 1.31 & 0.1 & -0.9 \\
\hline $1.0 \mathrm{~Hz}, 3 \mathrm{~dB}$ & 4 elements & Yes & 365 & 2.05 & 1.65 & 0.6 \\
\hline
\end{tabular}

TABLE II

Maximum mean carried traffic and maximum number of mobile users that can be supported by the network, whilst meeting the conservative quality constraints. The carried traffic is expressed in terms of normalised Erlangs (Erlang $/ \mathrm{km}^{2} / \mathrm{MHz}$ ) for the network described in Table I both with and without beamforming (as well as with and without independent up/down-link beamforming), and also with and without shadow fading having a standard deviation of $3 \mathrm{~dB}$ for $\mathrm{SF}=16$.

ploying beamforming using two and four element arrays is given in Table II, along with the teletraffic carried and the mean mobile as well as base station transmission powers required.

\section{REFERENCES}

[1] R. Prasad, CDMA for Wireless Personal Communications. Artech House, Inc., 1996.

[2] R. Steele and L. Hanzo, Mobile Radio Communications. IEEE Press John Wiley, 2nd ed., 1999.

[3] T. Ojanperä and R. Prasad, Wideband CDMA for Third Generation Mobile Communications. Artech House, Inc., 1998.

[4] J.S. Blogh, L. Hanzo: The Network Performance of Multi-rate FDD-mode UMTS, VTC 2001 Spring, Rhodes, Greece, 6-9 June, 2001

[5] S. Verdú, Multiuser Detection. Cambridge University Press, 1998.

[6] M. M. L. Cheng and J. C. I. Chuang, "Performance evaluation of distributed measurement-based dynamic channel assignment in local wireless communications," IEEE JSAC, vol. 14, pp. 698-710, May 1996.

[7] J.S. Blogh, P.J. Cherriman, L. Hanzo: Dynamic Channel Allocation Techniques Using Adaptive Modulation and Adaptive Antennas, IEEE JSAC, February, 2001, Vol. 19, No. 2, pp 312-321

[8] J.S. Blogh, L. Hanzo: IMT 2000 and Intelligent Wireless Networking Smart Antennas and Adaptive Modulation, John Wiley, to appear

[9] H. Holma and A. Toskala, eds., WCDMA for UMTS : Radio Access for Third Generation Mobile Communications. John Wiley \& Sons, Ltd., 2000.

[10] R. Owen, P. Jones, S. Dehgan, and D. Lister, "Uplink WCDMA capacity and range as a function of inter-to-intra cell interference: theory and practice," in IEEE Proceedings of Vehicular Technology Conference, vol. 1, (Tokyo, Japan), pp. 298-303, 2000. 